\title{
Why should we switch chest compression providers every 2 minutes during cardiopulmonary resuscitation?
}

\author{
SANG DAE LEE*, JUN YOUNG HONG ${ }^{*}$ JE HYEOK OH \\ Department of Emergency Medicine, College of Medicine, Chung-Ang University, Seoul, Republic of Korea \\ ${ }^{*}$ These authors contributed equally to this manuscript as first authors. \\ Correspondence author \\ Je Hyeok Oh \\ Department of Emergency Medicine \\ College of Medicine, Chung-Ang University \\ 84 Heukseok-ro, Dongjak-gu, 06974 Seoul, Republic of Korea \\ Phone/Fax: 82-2-6299-1820, 82-2-6299-2558 \\ E-mail: jehyeokoh@cau.ac.kr
}

\section{ABSTRACT}

Objective. This study was conducted to determine whether trained male rescuers could maintain adequate chest compression depth (CCD) for longer than the current recommended guidelines of 2 minutes. Methods. Forty male medical doctors administered a 5-minute single rescuer cardiopulmonary resuscitation (CPR) to a manikin on the floor with conventional CPR or randomly administered continuous chest compressions (CCC). The ratio of compression to ventilation was set to 30:2 with mouth-to-mouth technique during conventional CPR. Chest compression data were recorded with an accelerometer device and divided into 1-minute segments for analysis.

Results. Although average CCD maintained the recommended depths throughout 5 minutes in conventional CPR, it decreased significantly with CCC (1 minute: $55.4 \pm 4.5 \mathrm{~mm}$; 2 minutes: $54.2 \pm 5.4 \mathrm{~mm}$; 3 minutes: $52.6 \pm 5.6 \mathrm{~mm}$; 4 minutes: 51.6 $\pm 5.5 \mathrm{~mm}$; 5 minutes: $49.9 \pm 5.8 \mathrm{~mm}, \mathrm{p}<$ $0.001)$. The average chest compression numbers $(\mathrm{ACCN})$ per minute were maintained over $80 / \mathrm{min}$ and have not been changed significantly within 5 minutes in the CCC. However, it didn't reach to the $80 /$ min and decreased significantly after 3 minutes compared to the baseline ACCN during first 1-minute segment in the conventional CPR.

Conclusions. Despite the chest compression providers being limited to trained male medical doctors, the average CCD decreased significantly within 5minutes with CCC. Although maintaining adequate CCD, ACCN in each minute decreased significantly after 3 minutes in the convention- al CPR. Therefore, we should rotate chest compression providers every 2 minutes regardless of the rescuer's qualifications and CPR methods.

Key words: cardiopulmonary resuscitation, mouth-to-mouth resuscitation, cardiac arrest, healthcare provider

\section{INTRODUCTION}

The 2015 cardiopulmonary resuscitation (CPR) guidelines recommend that the chest compression providers should rotate every two minutes or sooner. (1)This is because chest compression depth (CCD) starts to deteriorate significantly after 1.5 to 2 minutes of continuous chest compressions (CCC). (2) On the other hand, CCD decreases more slowly when the chest compression provider uses the conventional CPR method with a compression to ventilation ratio of 30:2. (3) Therefore, the chest compressor could maintain adequate CCD for a longer period with conventional CPR. However, it has not yet been revealed how long we can maintain adequate CCD with conventional CPR. In addition, chest compression quality could be affected by many factors, such as rescuer's gender, body weight, qualifications, and muscular fitness. (4-8) If we could restrict the chest compression provider to trained male rescuers, the chest compression quality might be improved. We hypothesized that trained male rescuers could maintain adequate $\mathrm{CCD}$ for longer than the current recommended guidelines of 2 minutes. This study was conducted to confirm our hypothesis in two different CPR methods: conventional CPR and CCC.

\section{METHODS}

Study design

This was a prospective randomized trial comparing the quality of chest compression between CCC and conventional CPR performed by trained male rescuers. The trial design was approved by the Institutional Review Board of our hospital. All participants gave their written informed consents before the trial.

\section{Study setting}

The trial was conducted using an adult cardiac arrest simulation. The simulation scenario assumed that a visitor to our hospital suddenly collapsed in a hospital corridor. Therefore, the simulation was conducted on the floor. A Little Anne (Laerdal Medical, Stavanger, Norway) was used as a simulated cardiac arrest patient. The study participant assumed the role of a bystander performing single rescuer CPR until arrival of the CPR team. The simulation time was set at 5minutes considering the average CPR team activation time.

As study participants, we recruited male medical doctors from our hospital's CPR team who had completed a basic life support course and an advanced cardiovascular life support course within the last 2 years. The participants were randomly allocated to group A or B. The participants of group A performed a 5-minute conventional CPR and the participant of group $\mathrm{B}$ performed a 5-minute CCC in the same setting. The mouth-to-mouth technique with a face shield was used as a ventilation method during conventional CPR. The chest compression to ventilation ra- 
tio was set to $30: 2$ according to the 2015 CPR guidelines. (9) The compression parameters were collected using CPRmeter (Laerdal Medical) and the data were divided into 1-minute segments for analysis by using Q-CPR Review V.3.1. software (Laerdal Medical).

\section{Statistical analysis}

The sample size was calculated using an average CCD as a primary outcome variable. We hypothesized that the average CCD of CCC was as much as $10 \%$ lower than that of the conventional CPR. In a previous study, the average CCD was $53.9 \pm 5.8$ $\mathrm{mm}$. (10) We set the two-sided significance level at 0.05 , the power of the test at $80 \%$, and the allowable difference at $5.39 \mathrm{~mm}$. The minimum number of participants was determined as 19 in each group using a web program (sample size calculator: two parallel-sample means). (11)

All statistical analyses were performed using IBM SPSS Statistics, version 23.0 (IBM Corp., Armonk, NY, USA). The continuous variables were presented as the mean \pm standard deviation (SD), and the categorical variables expressed as percentages. The Friedman test was used to determine whether the average chest compression parameters in 1-minute segments changed significantly for 5 minutes. The Mann-Whitney U-test was used to compare data between two groups. In addition, the Wilcoxon signed-rank test was used for statistical comparisons in average chest compression numbers (ACCN) per minute over consecutive 1-minute segments compared to baseline ACCN during the first 1 -minute segment. A p value $<0.05$ was considered statistically significant.

\section{RESULTS}

A total of 40 male medical doctors were recruited and randomly assigned to Group A or B. There were no significant differences in the baseline characteristics between the two groups (table 1).

Comparisons of chest compression parameters during 5 minutes

Both groups achieved adequate CCD during 5minutes without significant difference (Group A: $54.7 \pm 4.7 \mathrm{~mm}$, Group B: 52.6 $\pm 5.0 \mathrm{~mm}, \mathrm{p}=0.192$ ). The average chest compression rate was also adequate without significant difference (Group A: 116.0 \pm 11.8 , Group B: $116.4 \pm 8.9, p=0.883)$. As expected, total chest compression numbers between the two groups were significantly different (Group A: $376 \pm 35.9$ compressions, Group B: $585.4 \pm 44.1$ compressions, $\mathrm{p}<0.001)$. As a result, $\mathrm{ACCN}$ in each minute was significantly higher in the CCC than in the conventional CPR $(117.1 \pm 8.8$ vs. $75.2 \pm 7.2$ number $/ \mathrm{min}$, respectively, $\mathrm{p}$ $<0.001)$. However, average flow time in the conventional CPR group was greater than $60 \%$ and considered adequate by current standards $(64.8 \pm 4.0 \%)$. (9)
Changes in chest compression parameters over consecutive 1-minute segments

Although average CCD maintained the recommended depths throughout $5 \mathrm{~min}$ utes in conventional CPR ( 1 minute: 54.8 $\pm 5.3 \mathrm{~mm} ; 2$ minutes: $55.0 \pm 5.2 \mathrm{~mm} ; 3$ minutes: $54.9 \pm 5.2 \mathrm{~mm}$; 4 minutes: 54.7 $\pm 4.8 \mathrm{~mm}$; 5 minutes: $54.5 \pm 4.3 \mathrm{~mm}, \mathrm{p}=$ 0.974 ), it decreased significantly with CCC (1 minute: $55.4 \pm 4.5 \mathrm{~mm}$; 2 minutes: 54.2 $\pm 5.4 \mathrm{~mm} ; 3$ minutes: $52.6 \pm 5.6 \mathrm{~mm} ; 4$ minutes: $51.6 \pm 5.5 \mathrm{~mm}$; 5 minutes: 49.9 $\pm 5.8 \mathrm{~mm}, \mathrm{p}<0.001$, table 2). As a result, the average CCD in the CCC was significantly lower than that of the conventional CPR after 4-minutes (Group A: $54.5 \pm 4.3$ mm, Group B: $49.9 \pm 5.8 \mathrm{~mm}, \mathrm{p}=0.013$, figure1). The ACCN per minute were maintained over $80 / \mathrm{min}$ and have not been changed significantly within 5 minutes in the CCC ( 1 minute: $116.0 \pm 7.3 ; 2$ minutes: $117.2 \pm 8.1 ; 3$ minutes: $116.8 \pm 9.9 ; 4 \mathrm{~min}$ utes: $117.5 \pm 9.9 ; 5$ minutes: $117.3 \pm 10.5$, p $=0.553$, figure 2$)$. However, it didn't reach $80 / \mathrm{min}$ in the conventional CPR (1 minute: $79.3 \pm 11.5 ; 2$ minutes: $75.0 \pm 8.0 ; 3$ minutes: $74.8 \pm 9.8 ; 4$ minutes: $73.6 \pm 7.9$; 5 minutes: $74.6 \pm 9.1, \mathrm{p}=0.139$, figure 2 ). In addition, it decreased significantly after 3 minutes compared to the baseline ACCN during the first 1-minute segment in the conventional CPR ( 1 minute vs. 4 minute: $79.3 \pm 11.5$ vs. $73.6 \pm 7.9, \mathrm{p}=0.007 ; 1 \mathrm{~min}-$ ute vs. 5 minute: $79.3 \pm 11.5$ vs. $74.6 \pm 9.1$, $\mathrm{p}=0.036)$.

Table 1. Baseline characteristics of the study participants

\begin{tabular}{llll}
\hline Characteristics & Group A $(\mathbf{n}=\mathbf{2 0})$ & Group B $(\mathbf{n}=\mathbf{2 0})$ & p-value \\
\hline Age $($ year $)$ & $27.6 \pm 3.7$ & $27.6 \pm 3.0$ & 0.989 \\
\hline Height $(\mathrm{m})$ & $1.772 \pm 0.053$ & $1.752 \pm 0.062$ & 0.301 \\
\hline Weight $(\mathrm{kg})$ & $73.9 \pm 10.7$ & $76.2 \pm 8.0$ & 0.265 \\
\hline Body Mass Index $(\mathrm{kg} / \mathrm{m} 2)$ & $24.6 \pm 3.4$ & $26.5 \pm 3.1$ & 0.072
\end{tabular}

Data are presented as mean \pm SD

Table 2. Changes in average compression depth and average compression rate during the tests

\begin{tabular}{|c|c|c|c|c|c|c|}
\hline Parameters & $1 \mathrm{~min}$ & $2 \mathrm{~min}$ & $3 \mathrm{~min}$ & $4 \mathrm{~min}$ & $5 \mathrm{~min}$ & p-value \\
\hline \multicolumn{7}{|c|}{ Conventional cardiopulmonary resuscitation } \\
\hline Average CCD (mm) & $54.8 \pm 5.3$ & $55.0 \pm 5.2$ & $54.9 \pm 5.2$ & $54.7 \pm 4.8$ & $54.5 \pm 4.3$ & 0.984 \\
\hline Average CCR (numbers/minute) & $115.5 \pm 9.5$ & $116.2 \pm 11.2$ & $115.8 \pm 12.4$ & $116.4 \pm 12.6$ & $116.7 \pm 13.6$ & 0.721 \\
\hline \multicolumn{7}{|l|}{ Continuous chest compressions } \\
\hline Average CCD (mm) & $55.4 \pm 4.5$ & $54.2 \pm 5.4$ & $52.6 \pm 5.6$ & $51.6 \pm 5.5$ & $49.9 \pm 5.8$ & $<0.001$ \\
\hline Average CCR (numbers/minute) & $115.7 \pm 7.7$ & $116.4 \pm 8.1$ & $116.2 \pm 9.8$ & $116.8 \pm 9.9$ & $117.4 \pm 10.3$ & 0.498 \\
\hline
\end{tabular}

CCD, chest compression depth; CCR, chest compression rate.

$\mathrm{p}<0.05$ is presented in bold. 


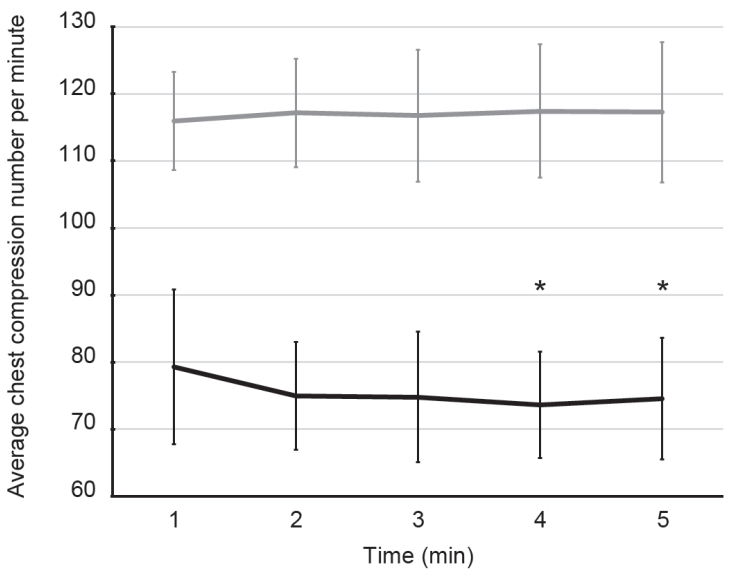

Figure 1. Changes in average chest compression depths calculated at 1-minute intervals in the two groups.

Black line: conventional cardiopulmonary resuscitation. Grey line: continuous chest compressions. The lines display the mean, and the error bars indicate \pm SD

${ }^{*} \mathrm{p}<0.05$.

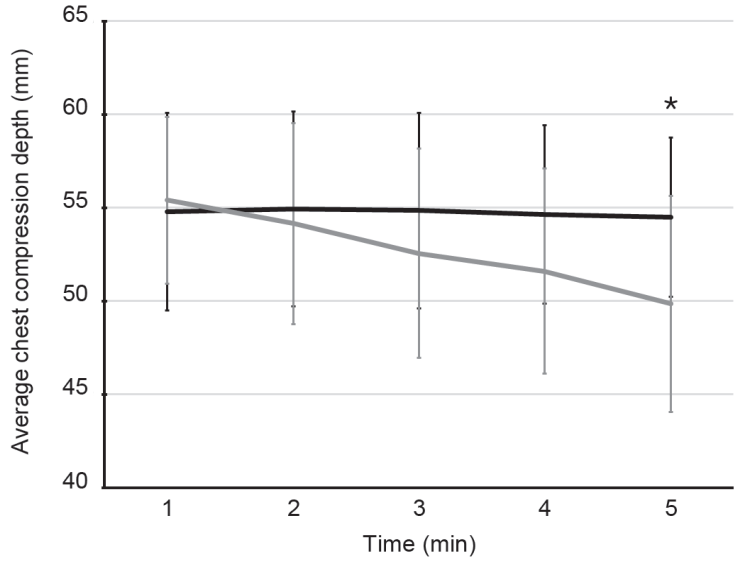

Figure 2. Changes in average chest compression numbers per minute over consecutive 1-minute segments compared to baseline average chest compression numbers during first 1-minute segment in the two groups.

Black line: conventional cardiopulmonary resuscitation. Grey line: continuous chest compressions. The lines display the mean, and the error bars indicate \pm SD.

${ }^{*} \mathrm{p}<0.05$.

\section{DISCUSSION}

We can improve survival after cardiac arrest by maintaining high-quality CPR (e.g., adequate rate, adequate depth, allowing full chest recoil, minimizing interruptions and avoiding excessive ventilation). (9) Therefore, we should try to achieve these goals during CPR.

Recently, many factors affecting quality of $\mathrm{CPR}$, especially adequate CCD and chest recoil have been identified. (5-7,12-19) In summary, the weight of the provider can impact the quality of CPR: being underweight has a negative effect on achieving adequate depth and being overweight has a negative effect on allowing full chest recoil. $(5,13,16)$ In addition, greater muscular strength has a positive effect on achieving adequate depth. $(6,7,14)$ If we can organize CPR teams that satisfy these conditions (e.g., limiting CPR team members to male rescuers who have normal body weight and greater muscular strength), we can improve the quality of CPR. (12)

This study was conducted to confirm whether we could maintain adequate CCD for a sufficient time by limiting chest compression providers to trained male medical doctors using two different CPR methods (Conventional CPR vs. CCC). Based only on the result of conventional CPR, it might seem that our hypothesis was true. Although the rescuers of the previous study did not maintain adequate CCD beyond 1minute with CCC, the study participant of our study (male medical doctors) maintained adequate CCD beyond 1minute with CCC. (8) However, CCD decreased significantly within 5 minutes even though we limited the chest compression providers to trained male medical doctors (figure $1)$.

By limiting chest compression providers to trained male medical doctors, we achieved an adequate chest compression fraction $(64.8 \pm 4.0 \%)$. Current guidelines recommend performing CPR with the goal of the chest compression fraction being as high as possible; the target should be at least $60 \%$. (9) However, the ACCN in each minute did not reach 80 throughout the 5 minutes with conventional CPR and decreased significantly after 3 minutes (figure 2). Previous study reported that the higher chest compression rates were significantly correlated with return of spontaneous circulation. (20) Average chest compression rates of the survivors were significantly higher than the non-survivors $(90 \pm 17$ versus $79 \pm 18$ compressions per minute). (20) Therefore, in our study, the ACCN in each minute during conventional $\mathrm{CPR}$ failed to reach the adequate compression number. In addition, significant decrease of the ACCN in each minute after 3minutes might be caused by the rescuer's accumulated fatigue.

The present study has several clinical implications. First, CCD decreased signifi- cantly within 5minutes with CCC despite limiting the chest compression provider to a trained male medical doctor. Therefore, we should rotate chest compression providers according to the current guidelines regardless of the rescuer's qualifications. Second, conventional CPR could not provide adequate chest compression numbers in each minute despite limiting the chest compression provider to a trained male medical doctor. In addition, ACCN in each minute decreased significantly after $3 \mathrm{~min}$ utes. Therefore, we should also rotate chest compression providers in the conventional CPR regardless of the rescuer's qualifications.

Our study had several limitations. First, the study design did not include CPR conducted by the trained female medical doctors or other types of rescuers. Therefore, our result could not confirm our hypothesis clearly; trained male rescuers could maintain adequate CCD for a longer period. Second, the data were measured using simulation models with manikins and accelerometer devices, and thus may have limited applicability to human patients. Third, the condition of the study participants was only restricted by gender. Although body weight and muscular strength of the rescuers could affect compression qualities, we could not control these conditions because of the small number of CPR team members. 


\section{CONCLUSIONS}

Despite the chest compression providers being limited to trained male medical doctors, the average CCD decreased signifi- cantly within 5 minutes with CCC. Despite maintaining adequate CCD, ACCN in each minute decreased significantly after 3 minutes in the conventional CPR. Therefore, we should rotate chest compression providers every 2 minutes regardless of the rescuer's qualifications and CPR methods.

\section{REFERENCES}

1. Link MS, Berkow LC, Kudenchuk PJ, Halperin HR, Hess EP, Moitra VK, et al. Part 7: Adult advanced cardiovascular life support: 2015 American Heart Association Guidelines Update for Cardiopulmonary Resuscitation and Emergency Cardiovascular Care. Circulation 2015; 132: S444-64.

2. Sugerman NT, Edelson DP, Leary M, Weidman EK, Herzberg DL, Vanden Hoek TL, et al. Rescuer fatigue during actual in-hospital cardiopulmonary resuscitation with audiovisual feedback: a prospective multicenter study. Resuscitation 2009; 80: 981-4.

3. Shin J, Hwang SY, Lee HJ, Park CJ, Kim YJ, Son YJ, et al. Comparison of CPR quality and rescuer fatigue between standard 30:2 CPR and chest compression-only CPR: a randomized crossover manikin trial. Scand J Trauma Resusc Emerg Med 2014; $22: 59$.

4. Lopez-Gonzalez A, Sanchez-Lopez M, Rovira-Gil E, Gonzalez-Garcia A, Ferrer-Lopez V, Martinez-Vizcaino V. Sex differences in the effort indicators during cardiopulmonary resuscitation manoeuvres on manikins. Eur J Emerg Med 2015; 22: 62-5.

5. Oh JH, Kim CW. Relationship between chest compression depth and novice rescuer body weight during cardiopulmonary resuscitation. Am J Emerg Med 2016; 34: 2411-3.

6. Lopez-Gonzalez A, Sanchez-Lopez M, Garcia-Hermoso A, Lopez-Tendero J, Rabanales-Sotos J, Martinez-Vizcaino V. Muscular fitness as a mediator of quality cardiopulmonary resuscitation. Am J Emerg Med 2016; 34: 1845-9.

7. Lin CC, Kuo CW, Ng CJ, Li WC, Weng YM, Chen JC. Rescuer factors predict high-quality CPR-a manikin-based study of health care providers. Am J Emerg Med 2016; 34: 20-4.

8. Hong DY, Park SO, Lee KR, Baek KJ, Shin DH. A different rescuer changing strategy between 30:2 cardiopulmonary resuscitation and hands-only cardiopulmonary resuscitation that considers rescuer factors: a randomised cross-over simulation study with a time-dependent analysis. Resuscitation 2012; 83: 353-9.

9. Kleinman ME, Brennan EE, Goldberger ZD, Swor RA, Terry M, Bobrow BJ, et al. Part 5: Adult basic life support and cardiopulmonary resuscitation quality: 2015 American Heart Association Guidelines Update for Cardiopulmonary Resuscitation and Emergency Cardiovascular Care. Circulation 2015; 132: S414-35.

10. Oh JH, Kim CW, Kim SE, Lee SJ, Lee DH. Comparison of chest compressions in the standing position beside a bed at knee level and the kneeling position: a non-randomised, single-blind, cross-over trial. Emerg Med J 2014; 31: 533-5.

11. Sample size estimation. Centre for Clinical Research and Biostatistics. The Chinese University of Hong Kong. Available from URL: http://www2.ccrb.cuhk.edu.hk/stat/Means.htm. accessed on June 22015.

12. Oh JH. A new strategy for cardiopulmonary resuscitation training. Commentary to the article: "The effect of strength training on quality of prolonged basic cardiopulmonary resuscitation" published in "Kardiologia Polska" 2017; 75, 1: 21-27. Kardiol Pol 2017; 75: 87-8.

13. Contri E, Cornara S, Somaschini A, Dossena C, Tonani M, Epis F, et al. Complete chest recoil during laypersons' CPR: Is it a matter of weight? Am J Emerg Med 2017. http://dx.doi.org/10.1016/j.ajem.2017.03.060.

14. Abelairas-Gómez C, Barcala-Furelos R, Szarpak Ł, García-García O, Paz-Domínguez Á, López-García S, et al. Strength training effects on quality of prolonged basic cardiopulmonary resuscitation. Kardiol Pol 2017; 75: 21-7.

15. Roh YS, Lim EJ. Factors influencing quality of chest compression depth in nursing students. Int J Nurs Pract 2013; 19: 591-5.

16. Krikscionaitiene A, Stasaitis K, Dambrauskiene M, Dambrauskas Z, Vaitkaitiene E, Dobozinskas P, et al. Can lightweight rescuers adequately perform CPR according to 2010 resuscitation guideline requirements? Emerg Med J 2013; 30: 159-60.

17. Hansen D, Vranckx P, Broekmans T, Eijnde BO, Beckers W, Vandekerckhove P, et al. Physical fitness affects the quality of single operator cardiocerebral resuscitation in healthcare professionals. Eur J Emerg Med 2012; 19: 28-34.

18. Ock SM, Kim YM, Chung J, Kim SH. Influence of physical fitness on the performance of 5-minute continuous chest compression. Eur J Emerg Med 2011; 18: 251-6.

19. Oh JH. A trade-off relationship between chest compression depth and chest wall recoil during cardiopulmonary resuscitation. Am J Emerg Med 2017; 35(10):1572-73. http://dx.doi.org/10.1016/j.ajem.2017.04.028.

20. Abella BS, Sandbo N, Vassilatos P, Alvarado JP, O’Hearn N, Wigder HN, et al. Chest compression rates during cardiopulmonary resuscitation are suboptimal: a prospective study during in-hospital cardiac arrest. Circulation 2005; 111: 428-34. 